BULL. AUSSTRAL. MATH. SOC.

05099

VOL. 19 (1978), 77-80.

\title{
Minimally locally \\ 1-connected graphs
}

\section{K.H. Kulkarni}

The local connectivity, $v k(G)$, of a graph $G$ is the minimum of the connectivities of neighbourhoods of the vertices of $G$. $G$ is minimally locally $n$-connected if $v k(G)=n$ and for every edge $x$ of $G, v k(G-x)=n-1$. A necessary and sufficient condition for a locally connected graph to be minimally locally 1 -connected is given, and it is shown that for $n \geq 7, \quad C_{n}^{2}$ is minimally locally 1 -connected.

\section{Introduction}

Our terminology is in conformity with that of Behzad and Chartrand [1]. For a vertex $v$ of a graph $G$, let $N(v)$ denote the set of all vertices of $G$ adjacent with $v$. The neighbourhood of $v$, denoted by $G(v)$, is the subgraph of $G$ induced by $N(v)$. $G$ is said to be locally connected if the neighbourhood of every vertex of $G$ is connected. $G$ is said to be locally n-connected if the neighbourhood of every vertex of $G$ is $n$-connected. The local connectivity, $v k(G)$, of $G$ is the maximum $n$ such that $G$ is locally $n$-connected. Similarly, $G$ is said to be locally n-edge connected if the neighbourhood of every vertex of $G$ is $n$-edge connected. The local edge-connectivity, $v k_{1}(G)$, of $G$ is the maximum $n$ for which $G$ is locally $n$-edge connected. Hence it follows that the local connectivity (edge-connectivity) of a graph is the minimum of the connectivities (edge-connectivities) of the neighbourhoods of its

Received 28 June 1978. This paper has non-empty intersection with the author's doctoral dissertation [3]. The author is most grateful to Dr E. Sampathkumar for his guidance. 
vertices. A graph may be locally connected without being connected, and conversely. For example, $2 K_{3}$ is locally connected while it is not connected; $K(1,3)$, which is connected is not locally connected. A graph is locally connected if and only if each of its components is locally connected.

The concept of local connectivity has been introduced by Chartrand and pippert [2]. Just like critically and minimally n-connected graphs, critically and minimally locally $n$-connected graphs can also be defined. A graph $G$ is critically locally $n$-connected if $v k(G)=n$ and for every vertex $v$ of $G, v k(G-v)=n-1 ; G$ is minimally locally n-connected if $v k(G)=n$ and $v k(G-x)=n-1$ for every edge $x$ of $G$. Just as the properties critically $n$-connected and minimally $n$-connected are independent in the sense that neither implies the other, so also are the properties critically locally n-connected and minimally locally $n$-connected. For example, the graph obtained from $K(n, n, n)$ by joining a pair of nonadjacent vertices is critically locally $n$-connected but it is not minimally locally $n$-connected; $K(n, n, n+1)$ is minimally locally $n$-connected while it is not critically locally $n$-connected. $K(n, n, n)$ is both critically and minimally locally $n$-connected. $K(n, n+1, n+1)$ is locally $n$-connected; but it is neither critically nor minimally locally $n$-connected.

The only critically 1 -connected graph is the complete graph of order 2. But critically locally I-connected graphs of many orders exist. In [4] a characterisation of such graphs is given. In this paper a necessary and sufficient condition for a locally connected graph to be minimally locally l-connected is given and it is shown that for $n \geq 7, c_{n}^{2}$ is minimally locally 1 -connected.

A whee $W$ is a cycle $C$ together with an additional vertex $w$ adjacent with every vertex of $C$. The cycle $C$ is called the circumcycle of $W$, and $W$ is called the wheel about $w$. If $P$ is a path in the cycle $C$, then $P$ is called a part of the wheel $W$. The connectivity and edge-connectivity of a graph $G$ will be denoted by $k(G)$ and $k_{1}(G)$, respectively. 


\section{Necessary and sufficient condition}

THEOREM. A locally connected graph $G$ is minimally locally 1-comected if and only if for every edge $x=u v$ of $G$ there exists $a$ vertex $w$ adjacent with both $u$ and $v$ such that $x$ is not a part of any wheel about $w$ in $G$.

Proof. Assume that $G$ is minimally locally l-connected. Consider an edge $x=u v$ of $G$. Let $H$ denote the graph $G-x$. Then $v k(H)=0$. Now, for every vertex $w^{\prime}$ of $G$ which is not adjacent with both $u$ and $v, H\left(w^{\prime}\right)=G\left(w^{\prime}\right)$; hence $k\left(H\left(w^{\prime}\right)\right)=k\left(G\left(w^{\prime}\right)\right) \geq 1$. Hence there exists a vertex $w$ adjacent with both $u$ and $v$ such that $k(H(w))=0$. Since $H(w)$ contains at least two vertices, namely, $u$ and $v$, it is disconnected. Also, $H(w)=G(w)-x$ and $G(w)$ is connected imply that $x$ is a bridge of $G(w)$. Suppose $G$ contains a wheel $W$ about $w$ such that $x$ is a part of $W$. Then every vertex of the circumcycle of $W$ is in $G(w)$. The part of this cycle not containing the edge $x$ constitutes a $u-v$ path in $G(w)$. This implies that $x$ is not a bridge of $G(w)$, which is a contradiction. Hence $x$ is not a part of any wheel about $w$ in $G$.

Conversely, assume that $G$ satisfies the hypothesis of the theorem. Consider an edge $x=u v$ of $G$. Let $w$ be a vertex of $G$ adjacent with both $u$ and $v$ such that $x$ is not a part of any wheel about $w$ in $G$. Let $G-x$ be denoted by $H$. Then $H(w)=G(w)-x$. Suppose $H(w)$ is connected. Then there exists a path $P$ in $H(w)$ joining $u$ and $v$. This path together with the edge $x$ constitutes a cycle $C$ every vertex of which is adjacent with $w$ in $G$. Hence $x$ is a part of a wheel about $w$ in $G$ which is a contradiction. Hence $H(w)$ is disconnected. Therefore $k(H(w))=0$. Now $H(w)=G(w)-x$ and $G(w)$ is connected imply that $x$ is a bridge of $G(w)$. Hence $v k_{1}(G)=1$, and hence $\dot{v k}(G)=1$. This proves that $G$ is minimally locally 1-connected.

The following corollary is immediate.

COROLLARY 1. If $G$ is a locally connected graph not containing a wheel, then $G$ is minimally locally 1-connected.

COROLLARY 2. For $n \geq 7, c_{n}^{2}$ is minimally locally l-connected. 
Proof. Let $n \geq 7$ be arbitrary and $c_{n}: v_{0}, v_{1}, \ldots, v_{n-1}, v_{0}$. Let $C_{n}^{2}$ be denoted by $G$. For a vertex $v_{i}$ of $G$, $N\left(v_{i}\right)=\left\{v_{i-2}, v_{i-1}, v_{i+1}, v_{i+2}\right\}$, where the indices of the vertices are taken modulo $n$. Hence $G\left(v_{i}\right)=P_{3}$, for $n=0,1, \ldots, n-1$. Hence $G$ is locally connected. Also, since $n \geq 7, G$ does not contain any wheel. Hence, by Corollary $1, G$ is minimally locally l-connected.

\section{References}

[1] Mehdi Behzad and Gary Chartrand, Introduction to the theory of graphs (Allyn and Bacon, Boston, 1971).

[2] Gary Chartrand and Raymond E. Pippert, "Locally connected graphs", Časopis Pést. Mat. 99 (1974), 158-163.

[3] K.H. Kulkarni, "Some topics in graph theory" ( $\mathrm{PhD}$ thesis, Karnatak University, Dharwar, 1976).

[4] K.H. Kulkarni, "A characterization of critically locally I-connected graphs", submitted.

Department of Mathematics,

Victoria Jubilee Technical Institute,

Bombay,

India. 